\title{
Transplante Renal em Paciente Pediátrico com Associação de Vater. Relato de Caso *
}

\section{Renal Transplantation in Vater Association Patient. Case Report}

\author{
Antonio Paulo Nogueira Costa ${ }^{1}$; Carmem Narvaes Bello, TSA ${ }^{2}$
}

\author{
RESUMO \\ Costa APN, Bello CN - Transplante Renal em Paciente Pediátri- \\ co com Associação de Vater. Relato de Caso
}

\begin{abstract}
JUSTIFICATIVA E OBJETIVOS: A associação de Vater é uma alteração politópica que inclui várias má-formações, sendo a hipoplasia radial a alteração mais freqüentemente encontrada ao nascimento. O objetivo deste relato é apresentar caso de associação de Vater em criança submetida a transplante renal.

RELATO DO CASO: Paciente do sexo masculino, 11 anos, 23 $\mathrm{kg}$, Hbs positivo submetido a esofagostomia com 30 horas de vida. Desde os 7 anos realiza hemodiálise, atualmente através de cateter atrial, por falta de outras vias de acesso. Submetido aos 11 anos a transplante renal com doador cadáver, sem intercorrências. Diurese adequada ao término das anastomoses vasculares. Drenagem de hematoma da fossa ilíaca $D$ no primeiro dia de pós-operatório. Alta 21 dias após o transplante com função renal normal.
\end{abstract}

CONCLUSÕES: A associação de Vater é uma ocorrência extremamente rara e complexa e o presente relato prende-se à realização, pela primeira vez, de transplante renal com doador cadáver em criança portadora desse defeito congênito, cujo resultado foi inteiramente satisfatório.

Unitermos: ANESTESIA, Pediátrica; CIRURGIA, Urológica: transplante renal; DOENÇAS: sindrome de Vater

\author{
SUMMARY \\ Costa APN, Bello CN - Renal Transplantation in Vater Associa- \\ tion Patient. Case Report
}

BACKGROUND AND OBJECTIVES: Vater association is a polytopic disorder including several malformations of which radial hypoplasia is the most common observed at birth. This report aimed at describing a case of Vater association in a child submitted to renal transplantation.

CASE REPORT: Male patient, 11 years old, $23 \mathrm{~kg}$, Hbs positive, submitted to esophagostomy at 30 hours of life. Since 7 years of age patient is submitted to hemodialysis, currently via atrial catheter for the lack of other access ways. Renal transplantation with cadaver donor was performed without intercurrences with adequate diuresis after vascular anastomoses. Right iliac fossa hematoma was drained in the first postoperative day. Patient was discharged 21 days after transplantation with normal renal function.

CONCLUSIONS: Vater association is an extremely rare and complex event and this report aimed at describing for the first time a renal transplantation with cadaver donor in a child with this congenital defect, the result of which has been fully satisfactory.

Key Words: ANESTHESIA, Pediatric; DISEASES: Vater syndrome; SURGERY, Urologic: renal transplantation

\section{INTRODUÇÃO}

$E^{n}$ ntende-se por associação de Vater o conjunto de má-formações congênitas que ocorre em combinações variadas (ao menos duas). Essas apresentam grande comprometimento funcional manifestando-se já nos primeiros anos de vida ${ }^{1}$.

Para certas associações não casuais de má-formações, ainda não se tem determinado se o padrão seria de seqüência ou síndrome. Criou-se então para essa situação a expressão associação. Um exemplo clínico é a associação de Vater que inclui má-formação vertebral, atresia anal, defeitos cardiovasculares ${ }^{1}$, atresia esofágica com fístula traqueoesofági-

\footnotetext{
* Recebido do (Received from) CET/SBA da Faculdade de Medicina da Universidade de São Paulo, SP

1. Médico Assistente da Disciplina de Anestesiologia da FMUSP

2. Médica Assistente Doutora da Disciplina de Anestesiologia da FMUSP
}

Endereço para correspondência (Correspondence to)

Dra. Carmem Narvaes Bello

Rua Padre Pereira de Andrade, 545/182-A

05469-000 São Paulo, SP

Apresentado (Submitted) em 14 de março de 2002

Aceito (Accepted) para publicação em 22 de outubro de 2002

(c) Sociedade Brasileira de Anestesiologia, 2003 ca, hipoplasia radial e agenesia renal unilateral ${ }^{2}$. Ocorrem predominantemente em associação e com apresentação esporádica em famílias sem antecedentes ${ }^{3}$. Como o defeito radial é o mais freqüentemente visualizado ao nascimento, a pesquisa das outras anomalias é mandatória ${ }^{4}$.

Geneticamente, associação de Vater é considerada uma alteração politópica e, portanto, referente à blastogênese, diferenciando-se das monotópicas associadas mais comumente à organogênese ${ }^{5}$.

Embora predomine a incerteza quanto à origem das má-formações, se geneticamente causadas ou predispostas ${ }^{6}$, todas as estruturas envolvidas são geneticamente normais ${ }^{7}$, considerando-se que as partes secundariamente afetadas por elas (spina bifida) sejam geneticamente normais ${ }^{8}$. O objetivo do presente relato é apresentar um caso de anestesia para transplante renal em paciente pediátrico portador de associação de Vater.

\section{RELATO DO CASO}

Paciente do sexo masculino, 11 anos, $25 \mathrm{~kg}$, pardo, com sorologia positiva para hepatite B. Nascido a termo com $2070 \mathrm{~g}$, apresentou cianose com desconforto respiratório, sendo diagnosticada atresia de esôfago com fístula distal. Com 30 horas de vida, optou-se por esofagostomia e gastrostomia. 
Nesse período, detectou-se fusão de arcos costais, destrocardia e criptorquidia.

Com 4 anos de idade, foi submetido a esofagocoloplastia retroesternal com pedículo pré-gástrico. Aos 5 anos submeteu-se a exploração de testículos que não foram encontrados.

Com 7 anos deu entrada no pronto socorro do Instituto da Criança do Hospital das Clínicas da FMUSP, com quadro de anasarca e hipertensão arterial, sendo diagnosticada insuficiência renal crônica terminal. Foi iniciada diálise peritoneal intermitente por cateter de Tenckhoff. A investigação etiológica da insuficiência renal crônica terminal mostrou hidronefrose bilateral, formação cística em ambos os rins e espessamento vesical. O quadro clínico foi definido como associação de Vater.

Os exames de controle mostravam infecção urinária de repetição com refluxo grau 3 à direita e grau 1 à esquerda (grau I dilatação só até ureteres, grau II - dilatação de ambos os ureteres e também da pélvis renal, grau III - dilatação ureteropielocalicial) e pielonefrite crônica evidenciada por biópsia renal.

Aos 10 anos, tentou-se obtenção de fístula arteriovenosa sem sucesso. Na tentativa de colocação de cateter venoso tipo permicath para hemodiálise, suspeitou-se de trombose de veia subclávia direita, o que levou a optar-se por veia femoral que também mostrou-se inadequada. Trombose da veia ilíaca comum e femoral esquerda foram constatadas pelo Doppler, tendo sido realizada trombólise e anticoagulação com heparina de baixo peso molecular (20 mg/dia). Pela falta de cateter para diálise, foi submetido à colocação de cateter atrial por toracotomia.

Aos 11 anos, apresentou episódio de sudorese e hipotensão arterial com diagnóstico de derrame pericárdico, que foi drenado por punção. Nesse período, os novos achados de investigação mostraram atrofia cortical na tomografia computadorizada e padrão de síndrome de ausência no EEG.

Aos 11 anos, pesando $23 \mathrm{~kg}$, foi submetido a transplante renal com doador cadáver. Os exames laboratoriais apresentavam creatinina $=7,1 \mathrm{mg} / \mathrm{dl}$; uréia $=180 \mathrm{mg} / \mathrm{dl}$; hemoglobina $=9,6 \mathrm{~g} / \mathrm{dl}$, e os demais exames dentro da normalidade. A monitorização constou de ECG de três derivações (FC $=120$ bpm), oxímetro de pulso $\left(\mathrm{SpO}_{2}=99 \%\right)$ e pressão não invasiva $(P A=150 \times 90 \mathrm{mmHg})$. APVC ( $3 \mathrm{mmHg})$ foi obtida através de cateter atrial que o paciente já utilizava para diálise. A anestesia foi induzida com fentanil $(100 \mu \mathrm{g})$, rocurônio (20 $\mathrm{mg}$ ), propofol (80 mg), seguida de intubação traqueal. Foi instituída ventilação mecânica. A manutenção da anestesia foi realizada com isoflurano a $1 \%$ em oxigênio e ar (50\%) com doses suplementares de rocurônio e fentanil.

No decorrer do procedimento anestésico foram administrados $7 \mathrm{ml} . \mathrm{kg}^{-1} \cdot \mathrm{h}^{-1}$ de cristalóides e $20 \mathrm{ml}^{\mathrm{kg}} \mathrm{kg}^{-1}$ de concentrado de hemácias. Ao iniciar-se a anastomose do pedículo vascular infundiram-se $15 \mathrm{ml} . \mathrm{kg}^{-1}$ de manitol lentamente e 1,5 $\mathrm{mg} \cdot \mathrm{kg}^{-1}$ de furosemida em bolus. A anestesia durou 8 horas. Ao término do procedimento, o rim apresentava aspecto favorável, sendo a PA $=130 \times 70 \mathrm{mmHg}, \mathrm{FC} 90 \mathrm{bpm}, \mathrm{PVC} 9$ $\mathrm{mmHg}, \mathrm{Hb}=9,7 \mathrm{~g} / \mathrm{dl}$ e Ht$=28 \%$. O paciente foi então encami- nhado para UTI, onde permaneceu no pós-operatório imediato. No primeiro dia de pós-operatório, verificou-se a presença de hematoma na fossa ilíaca esquerda, tendo sido encaminhado ao centro cirúrgico onde foi submetido à drenagem. Apresentou diurese média de $2 \mathrm{ml} . \mathrm{kg}^{-1} \cdot \mathrm{h}^{-1}$ nos três primeiros dias de pós-operatório. Recebeu alta com função renal normal 21 dias após o transplante.

\section{DISCUSSÃO}

O paciente portador da associação de Vater, em virtude das má-formações já citadas, necessita de cuidados especiais no manuseio anestésico.

Em relação à monitorização, a história de tromboses repetidas impossibilita o acesso venoso central através das veias subclávia e femoral para monitorização da PVC e uso de drogas vasoativas. A monitorização da PVC e uso de drogas foram realizados através de cateter instalado no átrio direito por toracotomia e até então usado para diálise. Também não se indicou a punção de artéria radial, sendo a monitorização da pressão arterial obtida através de método não invasivo, com espaçamento, evitando-se compressões repetidas em curto período de tempo.

A hiperreatividade brônquica é outro motivo de atenção especial. Plano anestésico adequado deve ser mantido antes de qualquer manipulação das vias aéreas, seja intubação, aspiração ou extubação.

Durante o transplante renal nesses pacientes, o controle volêmico deve ser extremamente rigoroso. As alterações pulmonares (hiperreatividade, história pregressa de anasarca e derrame pericárdico) limitam o volume de soluções cristalóides e colóides a serem infundidas e os sinais clínicos (ausculta pulmonar) e parâmetros de monitorização (PVCe ECG) são de vital importância.

É interessante salientar que, na casuística do Hospital das Clínicas da FMUSP, onde já foram realizados mais de 3.000 transplantes renais, esse foi o primeiro caso cuja etiologia da IRCT foi por associação de Vater.

\section{Renal Transplantation in Vater Association Patient. Case Report}

Antonio Paulo Nogueira Costa, M.D., Carmem Narvaes Bello, TSA, M.D.

\section{INTRODUCTION}

Vater association is a set of congenital malformations occurring in different combinations (at least two). These present major functional impairment and appear during the first years of life ${ }^{1}$.

For some non-casual malformation associations it is still not known if the pattern would be sequence or syndrome. For such situation, the word association has been coined. Aclini- 
cal example is Vater association including vertebral malformation, anal atresia, cardiovascular deffects ${ }^{2}$, esophageal atresia with tracheo-esophageal fistula, radial hypoplasia and unilateral renal agenesis ${ }^{3}$. This malformation predominantly occurs in association and with sporadic presentation in families without previous history ${ }^{4}$. Since the radial defect is most commonly detected at birth, the investigation of other abnormalities is mandatory ${ }^{5}$.

Vater association is genetically considered a polytopic change, thus related to blastogenesis and different from monotopic change, more commonly associated to organogenesis ${ }^{6}$.

Although the uncertainty about malformations origin, whether genetic or predisposed ${ }^{7}$, all involved structures are genetically normal, considering that parts secondarily affected by them (spina bifida) are genetically normal ${ }^{8}$.

This report aimed at describing a case of anesthesia for renal transplantation in a Vater association pediatric patient.

\section{CASE REPORT}

Male mulatto patient, 11 years old, $25 \mathrm{~kg}$, with positive serology for hepatitis B. Born at term with $2070 \mathrm{~g}$, he presented cyanosis with respiratory distress being diagnosed as esophageal atresia with distal fistula. At 30 hours of life, patient was submitted to esophagostomy and gastrostomy, when costal arches fusion, dextrocardia and undescended testis were diagnosed.

At 4 years of age patient was submitted to retrosternal esophagocoloplasty with pre-gastric pedicle. At 5 years of age patient was submitted to exploration of testes which were not found.

At 7 years of age, patient was admitted to the First Aid Unit of Instituto da Criança, Hospital das Clinicas, FMUSP with anasarca and arterial hypertension, being diagnosed as end stage renal disease (ESRD). Intermittent peritoneal dialysis was started with Tenckhoff catheter. Etiologic chronic investigation showed bilateral hydronephrosis, cyst formation in both kidneys and vesical thickening. Clinical presentation was defined as Vater association.

Control exams showed repetition urinary infection with reflux grade 3 to the right and grade 1 to the left (grade 1 - dilatation of the ureters, grade 2 - dilatation of both ureters and renal pelvis, grade 3 - ureteropyelocaliceal dilatation) and chronic pyelonephritis evidenced by renal biopsy.

An arterial-venous fistula was unsuccessfully attempted at 10 years of age. In the attempt to insert an intravenous permicath catheter for hemodialysis there was a suspicion of right subclavian vein thrombosis, what led us to try the femoral vein which was also inadequate. Common iliac and left femoral vein thrombosis were evidenced by Doppler, and thrombolysis was performed together with anticoagulation with low molecular weight heparin $(20 \mathrm{mg} /$ day). Due to the lack of catheter for dialysis an atrial catheter was inserted via thoracotomy.

At 11 years of age patient presented an episode of sweating and arterial hypotension diagnosed as pericardial effusion drained by puncture. At that time, new findings have shown cortical atrophy at CT and absence syndrome pattern at EEG. At 11 years of age and weighing $23 \mathrm{~kg}$, patient was submitted to renal transplantation with cadaver donor. Lab tests showed: creatinine $=7.1 \mathrm{mg}$. $/ \mathrm{dl}$; urea $=180 \mathrm{mg} / \mathrm{dl}$; hemoglobin $=9.6 \mathrm{~g} / \mathrm{dl}$, and remaining tests were normal. Monitoring consisted of 3 leads ECG ( $\mathrm{HR}=120 \mathrm{bpm})$, pulse oximetry $\left(\mathrm{SpO}_{2}=99 \%\right)$ and noninvasive blood pressure $(\mathrm{BP}=150 \times 90$ $\mathrm{mmHg})$. CVP $(3 \mathrm{mmHg})$ was obtained by the atrial catheter already used for patient dialysis. Anesthesia was induced with fentanyl $(100 \mu \mathrm{g})$, rocuronium $(20 \mathrm{mg})$ and propofol $(80 \mathrm{mg})$, followed by tracheal intubation and mechanical ventilation. Anesthesia was maintained with $1 \%$ isoflurane in oxygen and air $(50 \%)$ with additional rocuronium and fentanyl doses. During the anesthetic procedure, $7 \mathrm{ml} . \mathrm{kg}^{-1} \cdot \mathrm{h}^{-1}$ cristalloyds and $20 \mathrm{ml}^{\mathrm{kg}} \mathrm{kg}^{-1}$ of red cells concentrate were administered. At beginning of vascular pedicle anastomosis, $15 \mathrm{ml} . \mathrm{kg}^{-1}$ mannitol were slowly infused together with $1.5 \mathrm{mg} \mathrm{kg}^{-1}$ bolus furosemide. Anesthesia lasted 8 hours.

At procedure completion kidney had a favorable aspect and $\mathrm{BP}=130 \times 70 \mathrm{mmHg}, \mathrm{HR}=90 \mathrm{bpm}, \mathrm{CVP}=9 \mathrm{mmHg}, \mathrm{Hb}=9.7$ $\mathrm{g} / \mathrm{dl}$ and $\mathrm{Ht}=28 \%$. Patient was then referred to the ICU where he remained during the immediate postoperative period. A left iliac fossa hematoma was observed in the first postoperative day, being the patient referred to the operating center for drainage. Mean diuresis was $2 \mathrm{ml} . \mathrm{kg}^{-1} \cdot \mathrm{h}^{-1}$ in the first 3 postoperative days. Patient was discharged with normal renal function 21 days after transplantation.

\section{DISCUSSION}

Due to above mentioned malformations, Vater association patients need special anesthetic care.

In terms of monitoring, previous history of repeated thrombosis prevents central venous access via subclavian and femoral veins to monitor CVP and vasoactive drugs administration. CVP monitoring and drug administration were achieved through a catheter installed in the right atrium by thoracotomy and already used for dialysis. Radial artery puncture was also not indicated and blood pressure monitoring was obtained via a non invasive method with intervals, avoiding repeated compression in short periods of time.

Bronchial hyper-reactivity is another reason for special attention. An adequate anesthetic depth should be maintained before any further airway manipulation, being intubation, aspiration or extubation.

Volume control during renal transplantation in those patients should be extremely rigorous. Pulmonary changes (hyper-reactivity, previous anasarca history and pericardial effusion) limit crystalloid and colloid solutions volume to be infused and clinical signs (pulmonary auscultation) and monitoring parameters (CVP and ECG) are critically important. It is worth stressing that in Hospital das Clinicasl, FMUSP, where more than 3000 renal transplantations have been performed, this was the first case where ESRD etiology was due to Vater association. 


\section{REFERÊNCIAS - REFERENCES}

01. Smith DW, Jones KL - Recognizable Patterns of Human Malformation: Genetic, Embryology and Clinical Aspects $3^{\text {rd }}$ Ed, Philadelphia, WB Saunders, 1982;303-305.

02. Friedman WF, Child JS - Congenital Heart Diseases in the Adult, em: Harrison TR, Isselbacher KJ - Harrison`s Principles of Internal Medicine. $13^{\text {rd }}$ Ed, New York: McGraw-Hill, 1994; 1031-1041.

03. Werner W, The Vater syndrome from the urologyc viewpoint. Urology, 1998:203-205.

04. Martinez Frias ML - Errors of morphogenesis and developmental field theory. Am J Med Genet, 1998:291-296 .

05. Hollity HM, Relton JES - Orthopedic Diseases, em: Anesthesia and Uncommon Pediatric Diseases. $3^{\text {rd }} \mathrm{Ed}$, Philadelphia, WB Saunders, 1987;361-377.

06. Merei J, Hasthorpe S, Farmer P et al - Relationship between esophageal atresia with tracheoesophageal fistula and vertebral anomalies in mammalian embryos. Pediatr Surg, 1998; 33:58-63.

07. Shepard TH - A Catalog of Teratogenic Agents. $5^{\text {th }}$ Ed, Baltimore, The John Hopkins University Press, 1986;507-511.

08. Maruyama K - Diphallia and the Vater association. J Urol, 1999;1681-1684.

\section{RESUMEN}

Costa APN, Bello CN - Transplante Renal en Paciente Pediátrico con Asociación de Vater. Relato de Caso

JUSTIFICATIVA Y OBJETIVOS: La asociación de Vater es una alteración politópica que incluye varias mal-formaciones siendo la hipoplasia radial la alteración más frecuentemente encontrada en el nacimiento. El objetivo de este relato es presentar caso de asociación de Vater en niño sometido a transplante renal.

RELATO DE CASO: Paciente del sexo masculino, 11 años, 23 $\mathrm{kg}$. Hbs positivo sometido a esofagostomia con 30 horas de vida. Desde los 7 anos realiza hemodiálisis, actualmente a través de catéter atrial, por falta de otras vías de acceso. Sometido a los 11 años a transplante renal con donador cadáver, sin interocurrencias. Diuresis adecuada al término de las anastomosis vasculares. Drenaje de hematoma de la fosa ilíaca $D$ en el primer día de pós-operatorio. Alta 21 días después del transplante con función renal normal.

CONCLUSIONES: La asociación de Vater es una ocurrencia extremamente rara y compleja y el presente relato se prende a la realización, por la primera vez, de transplante renal con donador cadáver en niño portador de ese defecto congénito cuyo resultado fue enteramente satisfactorio. 